Pathologe $2007 \cdot 28: 105-112$

DOI 10.1007/s00292-007-0901-2

Online publiziert: 9. Februar 2007

๑) Springer Medizin Verlag 2007

\author{
E. Bruder ${ }^{1}$. L. M. Terracciano ${ }^{1} \cdot$ E. Passarge $^{2} \cdot$ W. A. Meier-Ruge ${ }^{1}$ \\ ${ }^{1}$ Institut für Pathologie, Universität Basel, Schweiz \\ ${ }^{2}$ Institut für Humangenetik, Universitätsklinikum Essen
}

\title{
Enzymhistochemie des klassischen und des ultrakurzen Morbus Hirschsprung
}

Der M. Hirschsprung bildet die wichtigste kongenitale Innervationsstörung des Gastrointestinaltrakts. Er beruht auf einer Migrationsstörung der Neuralleistenvorläuferzellen und geht mit einem aganglionären Darmabschnitt variabler Länge einher.

Der M. Hirschsprung manifestiert sich typischerweise im Neugeborenen- oder Kleinkindesalter als intestinales Obstruktionssyndrom (Übersicht bei [19]). Chronische Obstipation, aufgetriebenes Abdomen mit röntgenologisch nachweisbarem Megakolon proximal des aganglionären Anteils und Neigung zu Enterokolitis kennzeichnen das typische Krankheitsbild. Bei Neugeborenen ist ein $\mathrm{Me}$ koniumileus häufig.

Die Erstbeschreibung erfolgte durch Harald Hirschsprung 1889 in Kopenhagen [9]. Unabhängig davon hat $1869 \mathrm{Ab}$ raham Jacobi von der Columbia University in New York die Krankheit beschrieben, ohne aber auf die Eigenständigkeit der Krankheit hinzuweisen [12]. Die totale kongenitale intestinale Aganglionose wurde erst 60 Jahre nach der klinischen Erstbeschreibung nachgewiesen $[27,28]$, wenngleich 2 frühere Arbeiten das Fehlen von Ganglien erwähnen, ohne deren Bedeutung zu ermessen $[4,26]$.

Die Therapie des M. Hirschsprung besteht gegenwärtig in der Resektion des aganglionären Darmabschnitts. Durch die chirurgische Intervention können etwa $75 \%$ aller Patienten geheilt werden. Dafür stehen mehrere Verfahren zur Verfügung, von denen beim klassischen M. Hirschsprung die Durchzugstechnik nach Soave oder De La Torre bevorzugt wird $[5,10$, $15,25]$.

Die Diagnose des M. Hirschsprung ist seit der Einführung der enzymhistochemischen Biopsiediagnostik eine Aufgabe der Pathologie [2, 17]. Die folgende Arbeit beschreibt die wesentlichen Grundprinzipien der Morphologie des M. Hirschsprung und die besonderen Aspekte seiner Unterformen.

\section{Embryogenese des enteralen Nervensystems}

Das morphologische Bild des M. Hirschsprung und seiner Unterformen erklärt sich aus der Embryogenese des enteralen Nervensystems $[1,11,24]$. Das enterale Nervensystem stammt von Zellen der Neuralleiste $a b$, welche in der frühen Embryonalperiode - während der 6. bis 12. Embryonalwoche - in kraniokaudaler Richtung in den Gastrointestinaltrakt migrieren $[6,13]$. Die Zellmigration folgt einer definierten zeitlichen Sequenz von rostral nach kaudal.

In der 5. Embryonalwoche wachsen Nervenfasern des extramuralen Parasympathikus aus den Sakralwurzeln $\mathrm{S}_{2}$ bis $\mathrm{S}_{4}$ in die Muscularis propria ein, noch bevor die Neuroblastenvorläuferzellen aus dem Halsparasympathikus eingewandert sind.
Bei der normalen Entwicklung des enteralen Nervensystems treten in der 6. Embryonalwoche Neuroblasten in Ösophagus und Magen auf, in der 8. Embryonalwoche sind Neuralleistenvorläuferzellen in den gesamten Dünndarm eingewandert, und die migrierenden Neuralleistenzellen erreichen mit der 12. Embryonalwoche die Analregion. Eine kritische Zeit scheint zwischen der 8. und 12. Woche zu liegen, wenn sich der distale Plexus bildet.

Es findet während dieser 4 Wochen eine modulierende Verschaltung zwischen den einwandernden Neuroblasten und den einwachsenden Nervenfasern statt.

Der submuköse Plexus wird von Neuroblasten gebildet, die vom Plexus myentericus durch die zirkuläre Muskelschicht in die Submukosa einwandern. Der submuköse Plexus wird ebenfalls in kaudaler Richtung gebildet, aber später, während des 3. und 4. Monats [24].

Erreichen die Neuralleistenvorläuferzellen ihren Bestimmungsort im Analring jedoch nicht, so resultiert ein distales aganglionäres Segment und damit ein M. Hirschsprung [6, 13]. Die Aganglionose betrifft sowohl Plexus myentericus als auch Plexus submucosus. Dieses aganglionäre Darmsegment ist stark kontrahiert und wirkt infolge fehlender Peristaltik obstruierend wie eine Stenose, sodass es zur Ausbildung eines Megakolons proximal des aganglionären Segments kommen kann. Zwischen Megakolon und dis- 


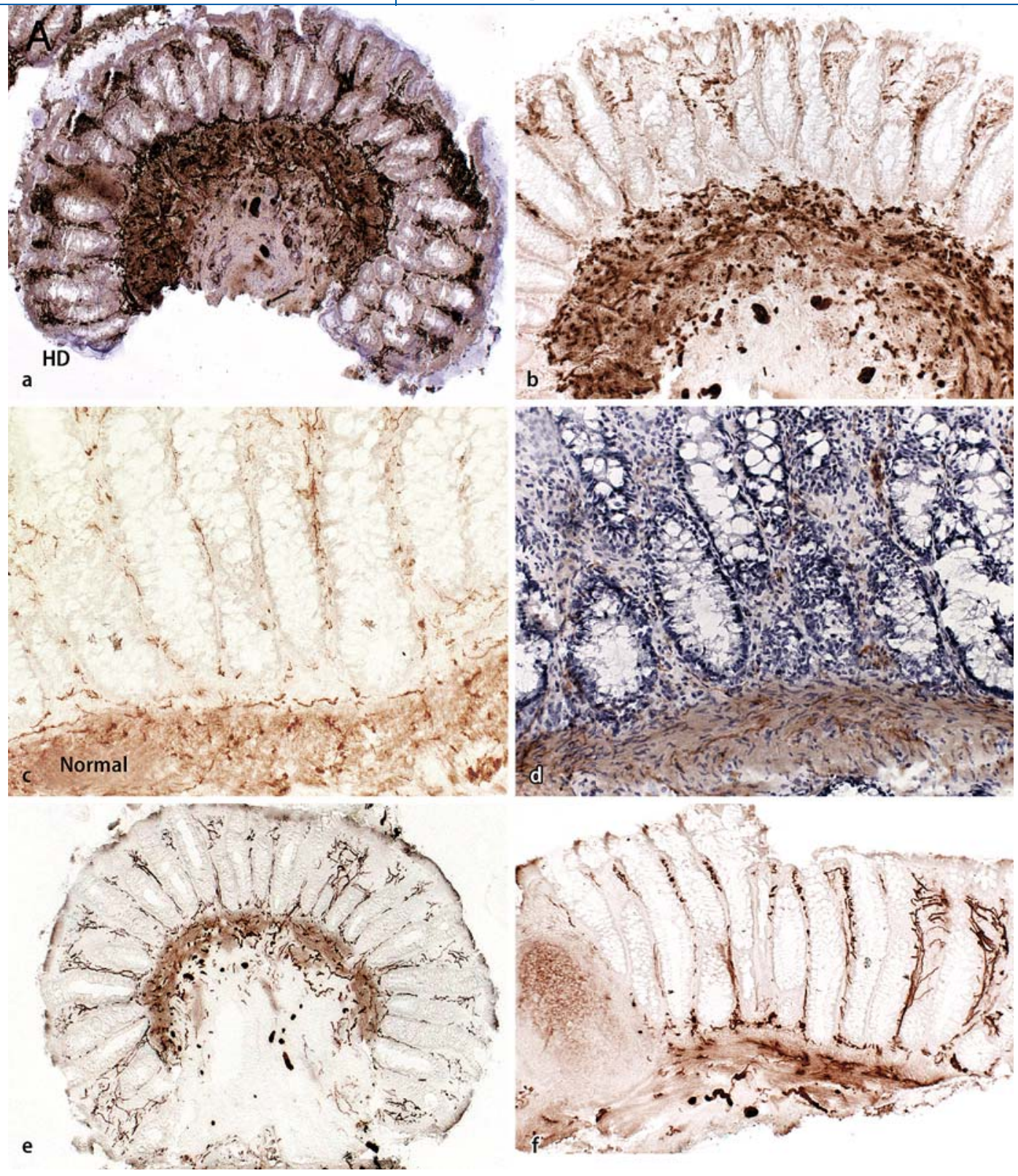

Abb. $1 \Delta$ Rektumschleimhautbiopsien. a Biopsie 3,5 cm ab ano bei einem 3-jährigen Jungen mit klassischem M. Hirschsprung mit massiv gesteigerter ACHE-Aktivität in den Nervenfasernetzen der Muscularis mucosae und in der Lamina propria mucosae. Die Steigerung der ACHE-Aktivität ist in der Hämalaun-gegengefärbten Enzymhistochemie für ACHE deutlich erkennbar (Vergr. 1:50). b Die Steigerung des Parasympathikotonus ist ohne Hämalaun-Gegenfärbung noch deutlicher erkennbar (Vergr. 1:100). c Biopsie $3 \mathrm{~cm}$ ab ano bei einem Vergleichspatienten mit normaler Innervation. Normale ACHE-Aktivität in den Nervenfasernetzen der Muscularis mucosae. Nur vereinzelte ACHE-positive Nervenfasern im Stroma der Lamina propria mucosae. Klare Beurteilbarkeit der niedrigen ACHE-Aktivität ohne Hämalaun-Gegenfärbung (Enzymhistochemie für ACHE; Vergr. 1:100). d Bei normaler Innervation besteht eine eingeschränkte Beurteilbarkeit der niedrigen ACHE-Aktivität bei Hämalaun-Gegenfärbung (Vergr. 1:100). e Biopsie $3 \mathrm{~cm}$ ab ano bei einem 3 Monate alten Jungen mit klassischem M. Hirschsprung. Deutlich gesteigerte ACHE-Aktivität in den Nervenfasernetzen der Muscularis mucosae mit Nachweis zahlreicher ACHE-positiver Nervenfasern im Stroma der Lamina propria. Deutlich erkennbare hypertrophe Nervenfaserbündel in der Submukosa (Enzymhistochemie für ACHE; Vergr. 1:50). f Rektumschleimhautbiopsie 3,5 cm ab ano bei einem Neugeborenen mit klassischem M. Hirschsprung: Ein Lymphfollikel verdrängt die ACHE -positiven Nervenfasern (Enzymhistochemie für ACHE; Vergr. 1:100) 
talem aganglionären Segment findet sich meist eine trichterförmige hypoganglionäre Übergangszone.

\section{Morphologisches Bild des klassischen Morbus Hirschsprung}

Fehlen beim M. Hirschsprung die Nervenzellen des Plexus myentericus im distalen Kolonsegment, so entfällt die Modulation der Aktivität der Nervenfasern des extramuralen Parasympathikus durch die Nervenzellen des Plexus myentericus. Es kommt zur permanenten AcetylcholinSekretion des sakralen Parasympathikus mit simultaner Steigerung der Acetylcholinesterase- (ACHE-)Aktivität und in der Folge zum Hypertonus der Muscularis propria mit obstruierender Wirkung im aganglionären Segment. Die gesteigerte Acetylcholin-Sekretion und die damit einhergehende reaktive Steigerung der Acetylcholinesterase-Aktivität wird auch als gesteigerter Parasympathikotonus bezeichnet.

Da der Neurotransmitter Acetylcholin sofort im synaptischen Spalt inaktiviert wird, taugt dieses Molekül für die Diagnostik des M. Hirschsprung nicht. Hingegen ist die simultan mit der Acetylcholin-Ausschüttung stattfindende Erhöhung der Acetylcholinesterase-Aktivität noch über Stunden nachweisbar und daher für die histopathologische Diagnostik des M. Hirschsprung geeignet $[14,16$, $17,18]$.

Die Steigerung der Acetylcholinesterase-Aktivität lässt sich auch in der Schleimhautbiopsie des Rektums nachweisen. Im Vergleich weist die normale Schleimhaut eine lediglich geringe Acetylcholinesterase-Aktivität auf. Beim klassischen M. Hirschsprung finden sich mit starker Steigerung Acetylcholinesterase-positive Nervenfasernetze in der Muscularis mucosae und im Stroma der Lamina propria mucosae (• Abb. 1 a-f). Die gesteigerte Acetylcholinesterase-Aktivität ist natürlich auch in der Muscularis propria nachweisbar, aber zur Diagnose des M. Hirschsprung genügt der Nachweis der Acetylcholinesterase-Steigerung an der Schleimhautbiopsie.

Die enzymhistochemische Darstellung des gesteigerten Parasympathikotonus beim klassischen M. Hirschsprung erlaubt

Pathologe 2007 · 28:105-112 DOI 10.1007/s00292-007-0901-2

(c) Springer Medizin Verlag 2007

\section{E. Bruder - L. M. Terracciano - E. Passarge - W. A. Meier-Ruge Enzymhistochemie des klassischen und des ultrakurzen Morbus Hirschsprung}

Zusammenfassung

Der M. Hirschsprung bildet die wichtigste gastrointestinale Motilitätsstörung in der frühkindlichen pathologischen Diagnostik. Die fehlerhafte kraniokaudale Migration der aus der Neuralleiste stammenden Neuroblastenvorläuferzellen führt zu einem aganglionären Kolonsegment variabler Länge: Beim klassischen M. Hirschsprung (60-75\%) umfasst das aganglionäre Segment Rektum und Sigma, der ultrakurze M. Hirschsprung (5$10 \%$ ) ist auf die distalen $3-4 \mathrm{~cm}$ oder den unmittelbaren rektoanalen Übergang beschränkt.

Die myenterischen Ganglien des normalen enteralen Nervensystems modulieren die parasympathische Innervation der Sakralwurzeln S2 bis S4. Beim M. Hirschsprung entfällt diese Modulation. Der gesteigerte Parasympathikotonus führt im aganglionären Segment zu einer spastischen Pseudoobstruktion. Enzymhistochemisch lässt sich der gesteigerte Parasympathikotonus am na-

\section{Enzyme histochemistry of classical and ultrashort Hirschsprung's disease}

\section{Abstract}

Hirschsprung's disease is the most important type of gastrointestinal dysmotility in neonatal pathology. Aberrant craniocaudal migration of neural crest stem cells results in an intestinal aganglionic segment of variable length. In 'classical' Hirschsprung's disease ( $60-75 \%$ of cases), the aganglionic segment spans the rectum and sigma. Ultrashort Hirschsprung's disease (5-10\%) is restricted to the most distal 3-4 cm or immediate rectoanal transition only.

In the normal enteric nervous system, myenteric ganglia modulate the parasympathetic innervation of the sacral roots S2S4. The absence of myenteric ganglia in Hirschsprung's disease results in massively increased parasympathetic activity with abundant acetylcholine release and pseudo-obstruction in the aganglionic segment. This can be demonstrated in an enzyme histochemical reaction for acetylcholinesterase on tiven Kryostatschnitt in einer massiven Acetylcholinesterase-Reaktion darstellen und erlaubt an der Rektumschleimhautbiopsie die Diagnose eines klassischen M. Hirschsprung. Beim ultrakurzen M. Hirschsprung ist die gesteigerte Acetylcholinesterase-Aktivität nur in den Nervenfasernetzen der Muscularis mucosae und Submukosa nachweisbar, nicht aber in der Lamina propria mucosae. Die Gangliendichte im distalen rektoanalen Übergang ist physiologisch sehr gering. Das Fehlen von Ganglienzellen in einer Biopsie darf daher nicht zur (falschen) Diagnose eines ultrakurzen M. Hirschsprung verleiten. Diese ist ausschließlich enzymhistochemisch mit der Acetylcholinesterase möglich.

\section{Schlüsselwörter}

M. Hirschsprung · Ultrakurzer M. Hirschsprung · Enzymhistochemie · Acetylcholinesterase frozen sections, which is sufficient to diagnose the classical disease in rectal mucosal biopsies. In ultrashort Hirschsprung's disease, increased acetylcholinesterase activity is demonstrable only in nerve fibres of the muscularis mucosae and submucosa, but not the lamina propria mucosae. Submucosal and myenteric ganglia are physiologically scarce in the most distal rectum; absence of ganglia in a biopsy of the rectoanal transition must not be (wrongly) interpreted as ultrashort Hirschsprung's disease. Therefore, a diagnosis of ultrashort Hirschsprung's disease can be made exclusively using an enzyme histochemical reaction for acetylcholinesterase.

\section{Keywords}

Hirschsprung's disease - Ultrashort Hirschsprung's disease - Enzyme histochemistry · Acetylcholinesterase 

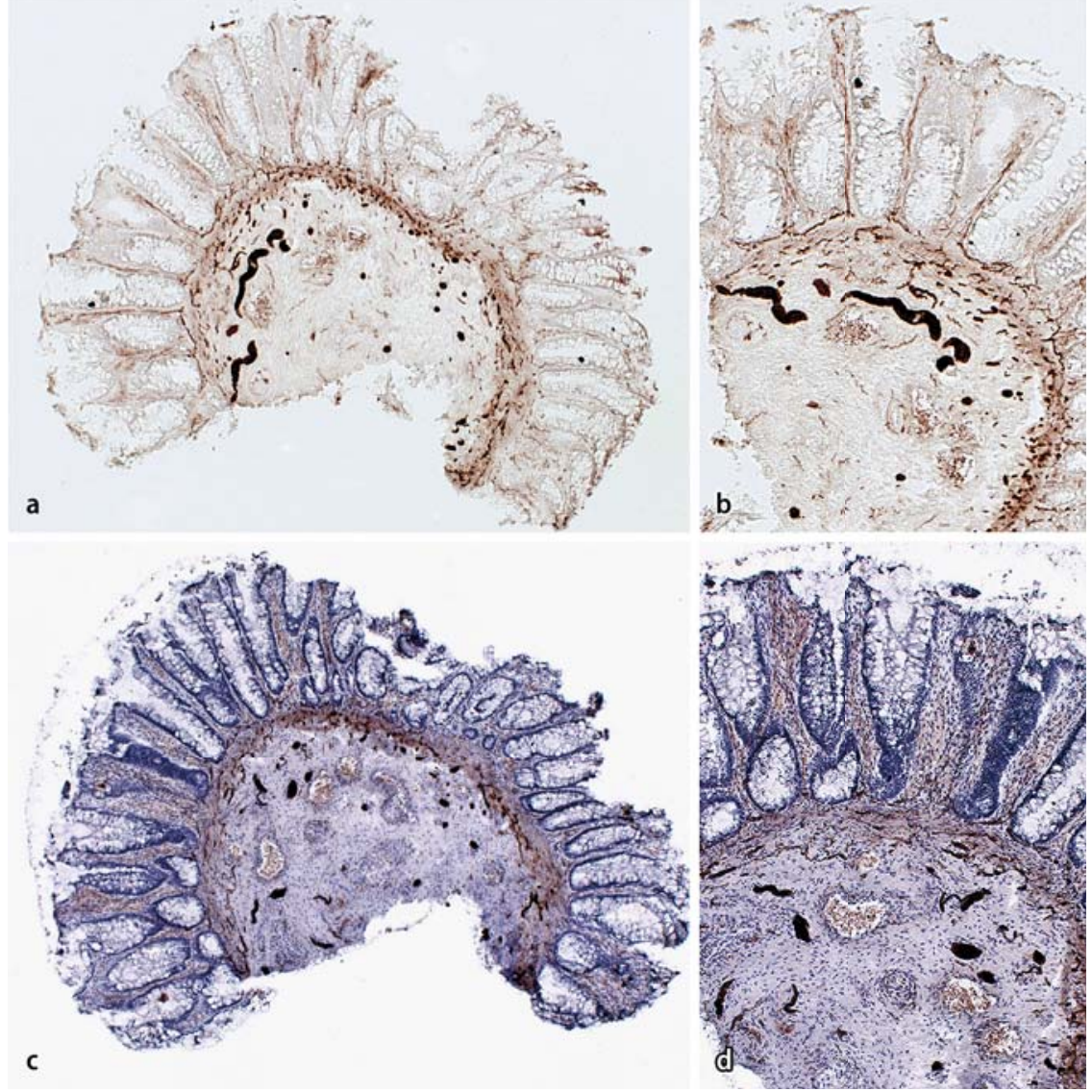

Abb. 2 A Ultrakurzer M. Hirschsprung. a,b Rektumschleimhautbiopsie $3 \mathrm{~cm}$ ab ano bei einem 3 Monate alten Jungen: Deutlich gesteigerte ACHE-Aktivität in den Nervenfasernetzen der Muscularis mucosae und deutlich hypertrophe Nervenfaserbündel der Submukosa. Kaum ACHE-positive Nervenfasern im Stroma der Lamina propria mucosae (Enzymhistochemie für ACHE; a Vergr. 1:50, b Vergr. 1:100). c,d Die deutlich gesteigerte ACHE-Aktivität in den Nervenfasernetzen der Muscularis mucosae und die deutlich hypertrophen Nervenfaserbündel der Submukosa sind auch in der Hämalaungegengefärbten ACHE-Reaktion klar erkennbar; hingegen schlechter beurteilbar die spärlichen ACHEpositiven Nervenfasern in der Lamina propria mucosae (Enzymhistochemie für ACHE mit HämalaunGegenfärbung; c Vergr. 1:50, d Vergr. 1:100)

die Diagnose an der Rektumschleimhautbiopsie auch dann, wenn keine Submukosa biopsiert wurde. Sie ist daher zur Sicherung des Verdachts auf einen M. Hirschsprung die Methode der Wahl und bildet weiterhin den Goldstandard der Diagnostik des M. Hirschsprung [3, 7, 20, 22].

\section{Morphologie des ultrakurzen Morbus Hirschsprung}

Der ultrakurze M. Hirschsprung bildet einen Sonderfall und umfasst eine Minderzahl von lediglich 5-10\% der Patienten. Er ist definiert als Aganglionose des distalsten Abschnittes des Gastrointestinaltrakts bis zu einer Ausdehnung von maximal 3-4 cm Länge $\mathrm{ab}$ ano. Die Aganglionose kann bei gleicher Symptomatik aber auch lediglich auf den distalen Sphincter inter- nus und/oder den M. corrugator cutis ani beschränkt sein.

Der ultrakurze M. Hirschsprung zeigt ein charakteristisches morphologisches Bild, welches im Gegensatz zu dem des klassischen M. Hirschsprung steht. Beim ultrakurzen M. Hirschsprung finden sich Nervenfasergeflechte mit einer gesteigerten Acetylcholinesterase-Aktivität lediglich in der Lamina muscularis mucosae und der Submukosa, nicht aber in der Lamina propria mucosae, wie dies beim klassischen M. Hirschsprung der Fall ist (• Abb. 2 a-d).

Ist die Aganglionose auf den Analring (M. corrugator cutis ani) beschränkt, so finden sich Nervenfasern mit stark erhöhter AcetylcholinesteraseAktivität nur im M. corrugator cutis ani (- Abb. 3 a-d). Zur Diagnose eines ul- trakurzen M. Hirschsprung ist also eine Biopsie aus dem unmittelbaren rektoanalen Übergang (Linea dentata) notwendig ( $\bullet$ Abb. 3 a,b). Hierbei sollte ein Ausläufer der distalen Sphinktermuskulatur miterfasst werden, um die Diagnose eines auf den Sphinkter beschränkten ultrakurzen M. Hirschsprung überhaupt stellen zu können (• Abb. 3 c,d).

Da physiologischerweise die Ganglien des Plexus myentericus und Plexus submucosus in den distalsten Zentimetern sehr spärlich sind, lässt sich ein ultrakurzer M. Hirschsprung ausschließlich an der enzymhistochemischen Reaktion für Acetylcholinesterase diagnostizieren [22]. Das Fehlen von Ganglien in einer Biopsie des rektoanalen Übergangs darf nicht mit einer Aganglionose gleichgesetzt werden. $\mathrm{Zu}$ beachten ist außerdem, dass eine $\mathrm{Bi}$ opsie zur Diagnose bzw. zum Ausschluss eines ultrakurzen M. Hirschsprung nicht nur Mukosa und Muscularis mucosae, sondern auch Submukosa enthalten sollte, da die stark Acetylcholinesterase-positiven Nervenfasern in der Lamina propria mucosae hier nicht wie beim klassischen M. Hirschsprung nachweisbar sind.

\section{Totale Aganglionose des Kolons}

Die totale Aganglionose stellt den zweiten Spezialfall dar, der bei der histologischen Diagnostik des M. Hirschsprung in Betracht gezogen werden muss. Sie ist selten und betrifft etwa 3-5\% der Patienten. Der aganglionäre Abschnitt des Plexus myentericus betrifft das gesamte Kolon und kann bis ins terminale Ileum reichen. Wie beim klassischen M. Hirschsprung resultiert im Rektosigmoid das Fehlen der myenterischen Modulation in einem massiv gesteigerten Parasympathikotonus mit einer enzymhistochemisch nachweisbaren Steigerung der Acetylcholinesterase-Aktivität der Nervenfasernetze der Muscularis mucosae und Muscularis propria.

Da die Innervation des sakralen Parasympathikus auf das Colon descendens, Sigma und Rektum beschränkt ist, lässt sich die Steigerung der Acetylcholinesterase-Aktivität nur dort nachweisen. Proximal des Innervationsbereiches des sakralen Parasympathikus, also im Bereich des Colon transversum, des Colon ascendens und des Zökums, ist trotz Agangli- 


\begin{abstract}
Abb. $3 \triangleright$ Biopsie aus der Linea dentata bei ultrakurzem M. Hirschsprung eines 4 Monate alten Jungen. a,b Deutlich gesteigerte ACHE-Aktivität in den Nervenfasernetzen von Ausläufern der Muscularis mucosae und des Analsphinkters (Enzymhistochemie für ACHE; a Vergr. 1:80, b Vergr. 1:200). c Deutlich gesteigerte ACHE-Aktivität in den Nervenfasernetzen der Ausläufer des Analsphinkters (Pfeil). Plattenepitheliale Überkleidung links, Mukosa mit Becherzellkrypten rechts (Enzymhistochemie für ACHE; Vergr. 1:80). d Deutlich erkennbarer Ausläufer des Analsphinkters in der Picro-Siriusrot-Färbung (Pfeil; Ver-
\end{abstract} gr. 1:80)
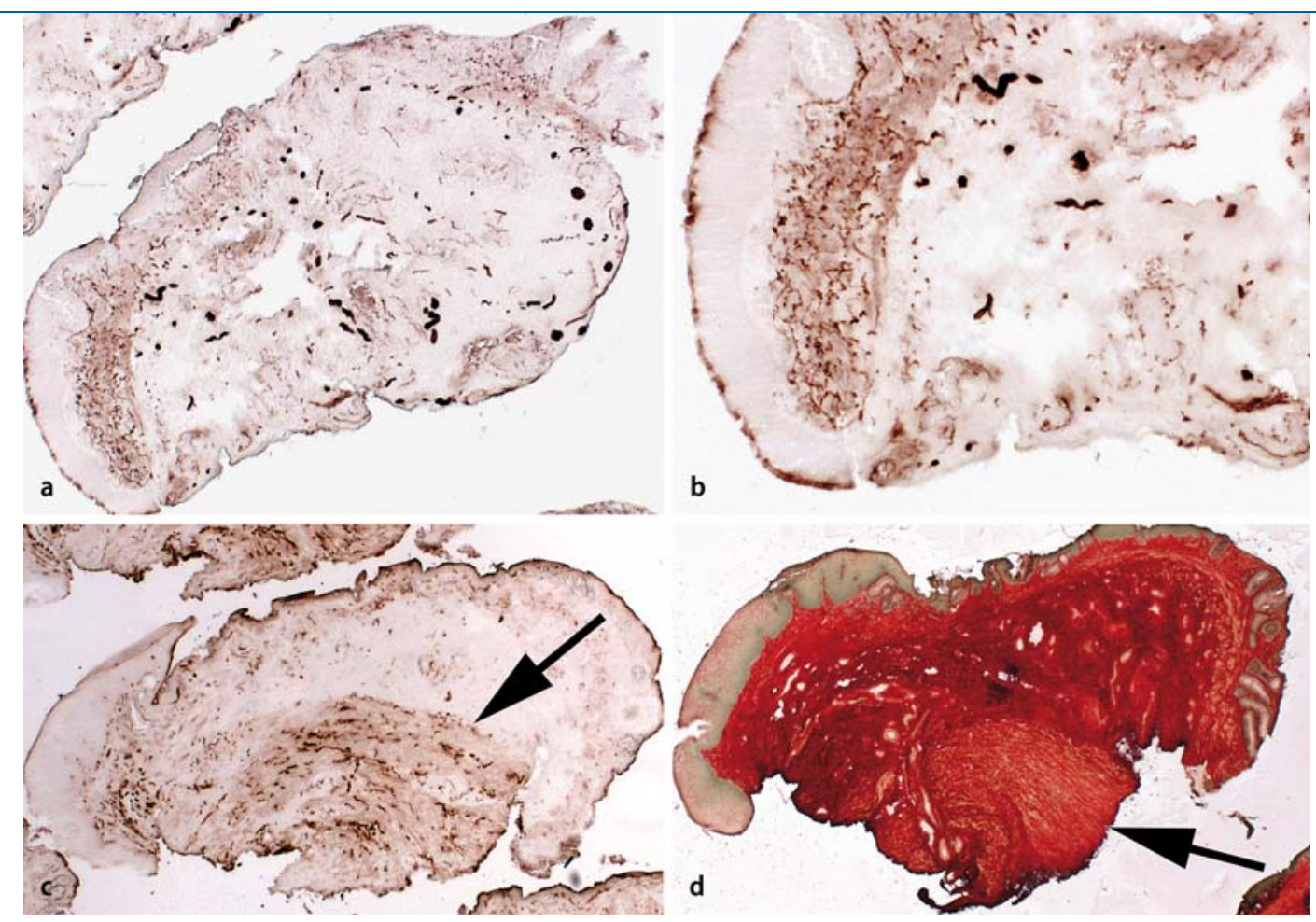

onose des Plexus myentericus in diesem Abschnitt bei der totalen Aganglionose des Kolons keine Steigerung der Acetylcholinesterase-Aktivität erkennbar [21].

Daher lässt sich die Diagnose der Aganglionose proximal der linken Kolonflexur nur aufgrund des totalen Mangels Acetylcholinesterase-positiver Strukturen in einer enzymhistochemischen Reaktion für Acelycholinesterase stellen. Hier ist bei Aganglionosen mit Ausdehnung proximal der linken Kolonflexur zusätzlich das Fehlen von Ganglien des Plexus myentericus an der Biopsie der Muscularis propria di- agnostisch hilfreich. Zur selektiven Darstellung der Nervenzellen der Ganglien der Plexus submucosus und myentericus ist die enzymhistochemische Reaktion für Laktatdehydrogenase am besten geeignet $[8,22]$.

\section{Hypoganglionäre Übergangszone}

Proximal des aganglionären Segments schließt sich meist eine 3-30 cm lange hypoganglionäre Übergangszone an. Zur Beurteilung der Gangliendichte im hypoganglionären Abschnitt dient die Dar- stellung des Zytoplasmas der Nervenzellen. Die enzymhistochemische Laktatdehydrogenase-Reaktion stellt selektiv die Ganglien des enteralen Nervensystems dar. Beim M. Hirschsprung sind in der Laktatdehydrogenase-Reaktion im aganglionären Abschnitt weder im Bereich des Plexus myentericus. noch des Plexus submucosus Ganglien oder Nervenzellen nachweisbar.

In der hypoganglionären Übergangszone lassen sich kleine, weit gestreute Ganglien mit wenigen, kleinkalibrigen Nervenzellen und langen interganglio-

\section{Hier steht eine Anzeige.}

\section{Springer}




\section{Schwerpunkt: Gastrointestinale Motilitätsstörungen}

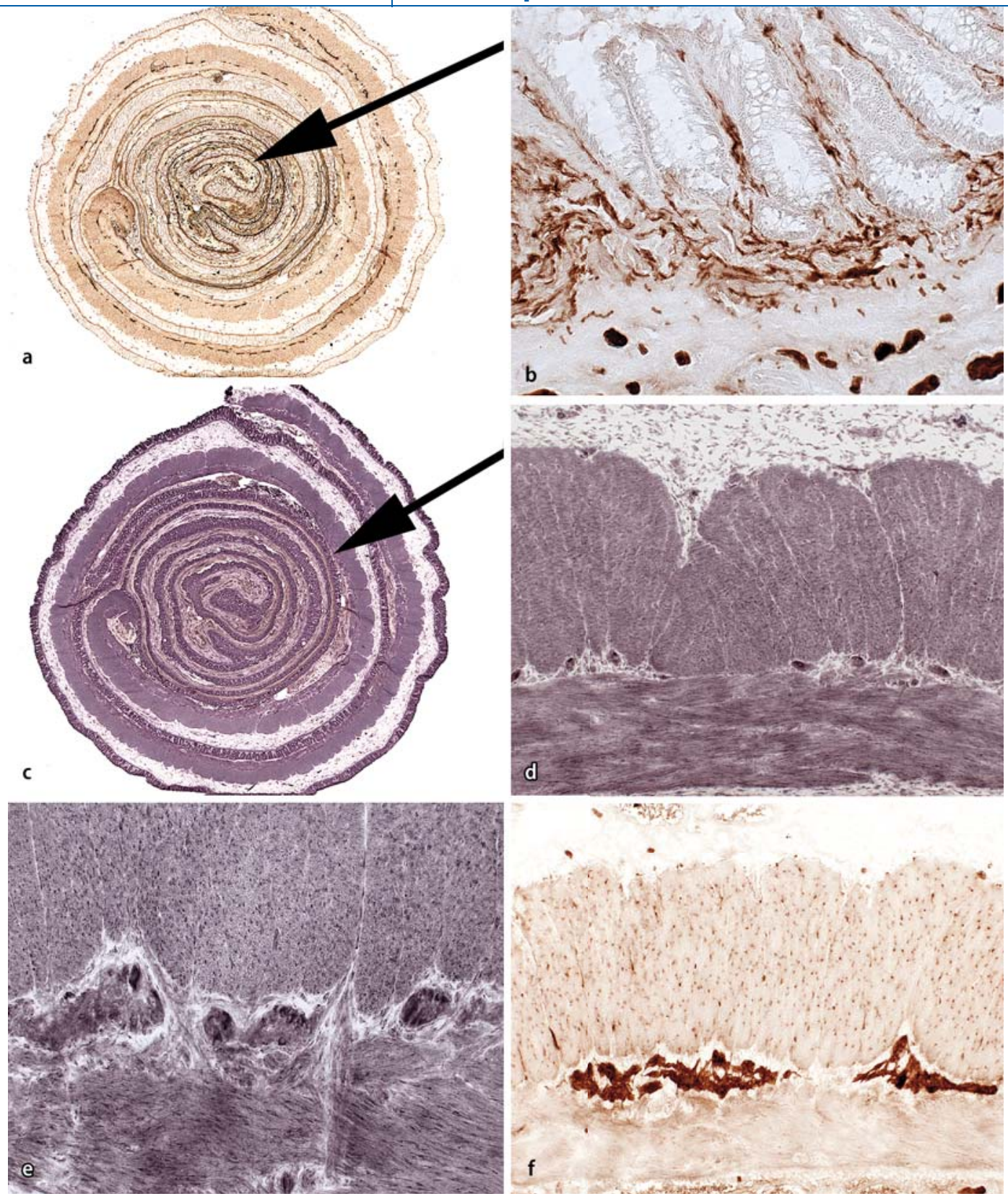

Abb. 4 A Rektosigmoidresektion nach Durchzugsoperation gemäß De la Torre bei klassischem M. Hirschsprung. Aufgerolltes Resektat von aboral (innen) nach oral (außen). Im Zentrum der Darmrolle der aborale Kolon-Mukosa-Schlauch. Die Muscularis propria verbleibt bei der Durchzugstechnik im Patienten (a Enzymhistochemie für ACHE und c für Laktatdehydrogenase; Lupenübersicht, Vergr. 1:2,5). a Aborales Resektatende im Zentrum der Darmrolle (Pfeil) im Bereich des aganglionären Segments: deutlich gesteigerte ACHE-Aktivität in den Nervenfasernetzen der Muscularis mucosae und im Stroma der Lamina propria mucosae (b, Enzymhistochemie für ACHE; Vergr. 1:200). c Hypoganglionäre Übergangszone in der Mitte der dargestellten Darmrolle (Pfei): verlängerte interganglionäre Abstände des Plexus myentericus, kleine nervenzellarme Ganglien (d, Enzymhistochemie für Laktatdehydrogenase; Vergr. 1:80). e Normal innervierter oraler Resektatrand: große nervenzellreiche Ganglien des Plexus myentericus mit geringen interganglionären Abständen (Enzymhistochemie für Laktatdehydrogenase; Vergr. 1:200). f Normal innervierter oraler Resektatrand: regelrechte ACHE-Aktivität der Nervenfasernetze der Muscularis propria (Enzymhistochemie für ACHE; Vergr. 1:80)

nären Abständen erkennen (• Abb. 4 af). Wird eine solche hypoganglionäre Übergangszone dokumentiert, sollte die chirurgische Resektion weiter proximal in einem Darmsegment mit regelhaftem Ganglienbesatz erfolgen, um ein postope- ratives Rezidiv der Obstipation oder sogar eines Megakolons zu vermeiden.

Bei der Hypoganglionose findet sich zwar eine typische schwache Acetylcholinesterase-Aktivität in den Nervenfasernetzen der Muscularis propria, diese ist je- doch für die Diagnose einer Hypoganglionose nicht ausreichend. Die Diagnose erfordert die Beurteilung der Ganglien in einer Dehydrogenase-Darstellung.

Die Swiss-Roll-Technik erlaubt eine hervorragende Übersicht über das rese- 
Abb. 5 - Neugeborener mit klassischem M. Hirschsprung (Enzymhistochemie für ACHE ohne HämalaunGegenfärbung).

a,b Rektumschleimhautbiopsie $3 \mathrm{~cm}$ ab ano. Deutlich gesteigerte $\mathrm{ACHE}-\mathrm{Ak}$ tivität der Nervenfasernetze der Muscularis mucosae und der Lamina propria mucosae. Hypertrophie der Nervenfaserbündel der Submucosa. Nebenbefundlich Hämolyseartefakte im Stroma der Lamina propria mucosae (a Vergr. 1:100, b Vergr. 1:200). c,d Rektosigmoidschleimhautbiopsie $10 \mathrm{~cm}$ ab ano. Wesentlich geringere Dichte ACHEpositiver Nervenfasern. Die Steigerung der ACHE-Aktivität ist ebenfalls deutlich ausgeprägt. Massiver Hämolyseartefakt in der Lamina propria mucosae (c Vergr. 1:100, d Vergr. 1:200)
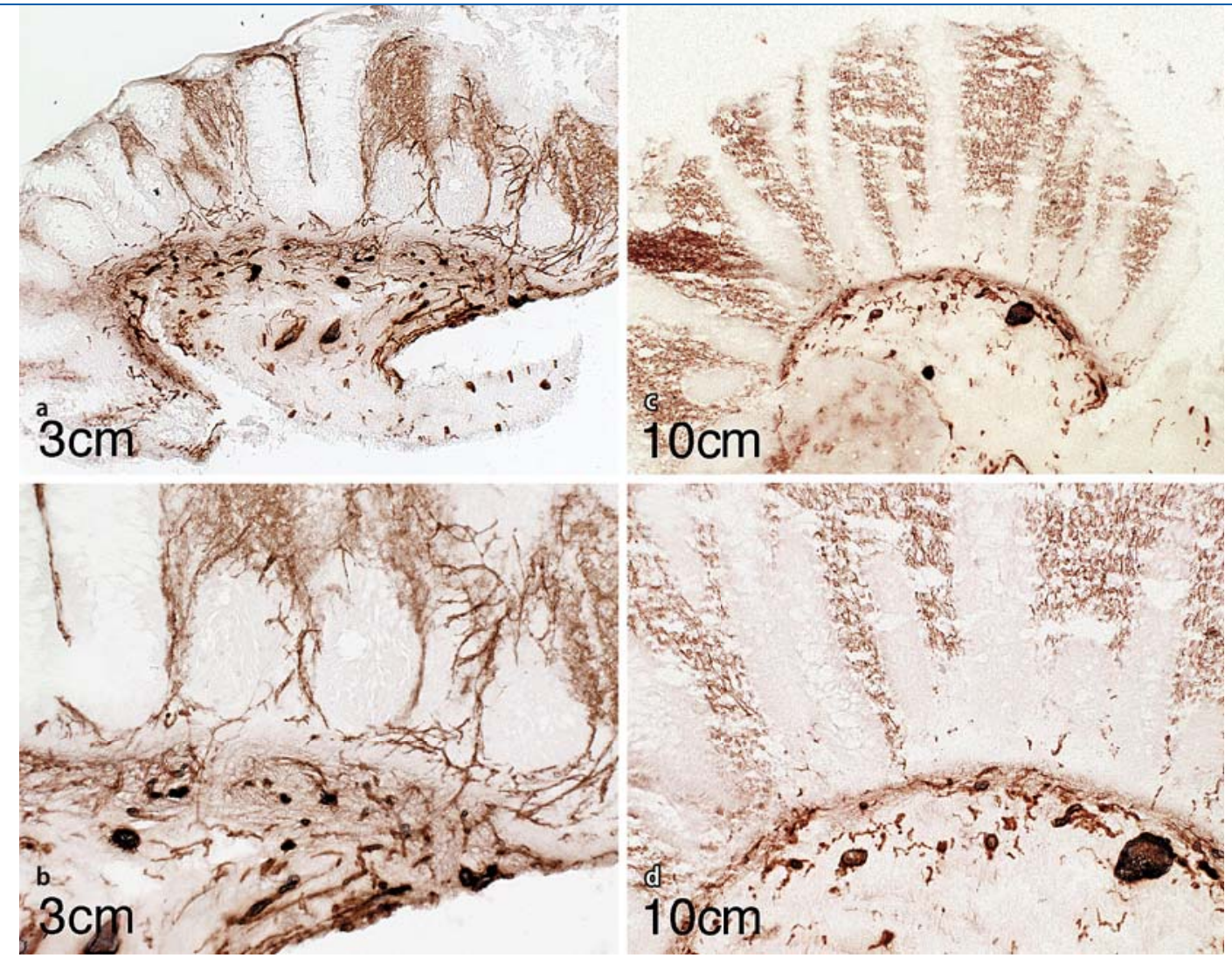

zierte Kolon. Pro Rolle lassen sich bis zu $15 \mathrm{~cm}$ Darm in einem einzigen Schnittpräparat überschauen. Dies erlaubt die optimale Einschätzung der Ausdehnung der hypoganglionären Übergangszone am Resektat (• Abb. 4 a-f).

\section{Intraoperative}

\section{Schnellschnittdiagnostik}

Da sich bei den meisten Patienten mit klassischem M. Hirschsprung eine hypoganglionäre Übergangszone proximal des aganglionären Segments findet und die Transportleistung des hypoganglionären Abschnitts im Allgemeinen nicht für eine suffiziente Peristaltik genügt, sollte die chirurgische Resektion die gesamte hypoganglionäre Übergangszone einschließen.

Die Durchführung eines intraoperativen Schnellschnittes ermöglicht dem Chirurgen eine vollständige Resektion der hypoganglionären Übergangszone. Die Qualität des Plexus myentericus lässt sich intraoperativ an mindestens $5 \mathrm{~mm}$ großen Biopsien der Muscularis propria mit Hilfe einer enzymhistochemischen Laktatdehydrogenase-Reaktion beurteilen. Die enzymhistochemische Laktatdehydrogena-
se-Reaktion zur Darstellung der Nervenzellen ist der HE-Färbung weit überlegen und daher auch am Schnellschnitt vorzuziehen.

Die intraoperative Information über Ausdehnung der hypoganglionären Übergangszone erlaubt dem Chirurgen die optimale Platzierung der Resektion unter Anwendung der am besten geeigneten chirurgischen Resektionstechnik.

Das chirurgische Resektat wird anschließend mit Hilfe der Swiss-Roll-Technik vollständig aufgearbeitet und die Ausdehnung der hypoganglionären Übergangszone am Längs- und Querschnitt bestätigt.

\section{Fehlerquellen in der morphologischen Diagnose}

Die enzymhistochemische Diagnose eines M. Hirschsprung basiert auf der Acetylcholinesterase-Aktivität der parasympathischen Nervenfasern des Kolons. Die Dichte der parasympathischen Nervenfasern nimmt von aboral nach oral ab, sodass weiter oral, z. B. $10 \mathrm{~cm}$ ab ano, das charakteristische Vollbild des klassischen M. Hirschsprung nicht mehr in typischer Weise ausgebildet ist (• Abb.5 a-d).
Die Biopsieentnahme bei Verdacht auf M. Hirschsprung sollte daher in einer Höhe von 1, 3 und $6 \mathrm{~cm}$ ab ano erfolgen und immer eine Biopsie aus der Linea dentata einschließen.

Lymphfollikel verdrängen die Nervenfasern in der Lamina propria und der Muscularis mucosae (• Abb. 1 f), sodass eine ausgeprägte lymphofollikuläre Hyperplasie das Risiko einer falschnegativen Diagnose birgt. Hämolyseartefakte in der Lamina propria mucosae, die sich als diffuse Färbungen darstellen und keine Nervenfasern erkennen lassen (• Abb. 5 a-d), sollten nicht zu falschpositiven Diagnosen verleiten.

Die konstante Schnittdicke von $15 \mu \mathrm{m}$ (Enddicke $4 \mu \mathrm{m}$ ) ist eine grundlegende Voraussetzung für eine zuverlässige Einschätzung der Acetylcholinesterase-Aktivität [23]. In einem Schnittpräparat von geringerer Schnittdicke kann die Acetylcholinesterase-Aktivität trotz eigentlicher Steigerung bei einer Aganglionose fälschlicherweise normal erscheinen und zu einer falsch-negativen Diagnose führen. Ein $4 \mu \mathrm{m}$ dicker Kyrostatschnitt hat eine Enddicke von etwa $1 \mu \mathrm{m}$.

Die Hämalaun-Gegenfärbung dient zwar der architektonischen Orientierung 
in der Schleimhautbiopsie, aber sie kann ebenfalls die Einschätzung der Acetylcholinesterase-Aktivität beeinträchtigen. Daher ist es ratsam, die AcetylcholinesteraseReaktion doppelt durchzuführen: einmal ohne Gegenfärbung zur exakten Einschätzung des Parasympathikotonus und einmal mit Hämalaun-Gegenfärbung zur architektonischen Übersicht $(\bullet$ Abb. 1 a-d).

\section{Fazit für die Praxis}

Die enzymhistochemische Untersuchung bildet weiterhin den Goldstandard in der Diagnostik des M. Hirschsprung. Das enzymhistochemische Bild der Subtypen des $M$. Hirschsprung ist abhängig von der Länge des aganglionären Segments und der Entnahmehöhe der Biopsie. Zur präoperativen Bestimmung der Ausdehnung eines aganglionären Segments sollten Stufenbiopsien mit Einschluss des rektoanalen Übergangs entnommen werden. Für den klassischen M. Hirschsprung sind Schleimhautbiopsien ausreichend.

Die intraoperative enzymhistochemische Schnellschnittdiagnostik erlaubt die Beurteilung des Plexus myentericus an einer ausreichend großen Biopsie der Muscularis propria aus dem Bereich des vorgesehenen Resektionsrandes. Eine Resektion durch eine hypoganglionäre, peristaltisch insuffiziente Übergangszone hindurch kann so vermieden werden. Fertig zusammengesetzte Reaktionskits sind erhältlich bei Districhem, Hohlweg 25, Ch-4104 Oberwil, E-Mail: districhem@bluewin.ch, oder Bio-Optica, Via San Faustina 58, I-20134 Milano, http:// www.bio-optica.it.

\section{Korrespondierender Autor \\ Prof. Dr. W. A. Meier-Ruge}

Institut für Pathologie, Universität Basel Schönbeinstraße 40, 4031 Basel, Schweiz meier-ruge@bluewin.ch

Interessenkonflikt. Es besteht kein Interessenkonflikt. Der korrespondierende Autor versichert, dass keine Verbindungen mit einer Firma, deren Produkt in dem Artikel genannt ist, oder einer Firma, die ein Konkurrenzprodukt vertreibt, bestehen. Die Präsentation des Themas ist unabhängig und die Darstellung der Inhalte produktneutral.

\section{Literatur}

1. Andrew A (1971) The origin of intramural ganglia. IV. The origin of enteric ganglia: a critical review and discussion of the present state of the problem. J Anatomy 108: 169-84

2. Borchard F, Meier-Ruge WA, Wiebecke B et al. (1991) Innervationsstörungen des Dickdarms: Klassifikation und Diagnostik. Pathologe 12: 171174

3. Coerdt W, Müntefering H, Rastorguev E, Gerein V (2004) Kongenitale Innervationsstörungen des Colon. Ein diagnostischer Leitfaden. Pathologe 25: 292-298

4. Dalla Valle A (1924) Contributo alle conoscenza della forma famigliare del megacolon congenito. Pediatria 32: 569-599

5. De La Torre-Mondragon L, Ortega-Salgado JA (1998) Transanal endorectal pull-through for Hirschsprung's disease. J Pediatr Surg 33: 12831286

6. Gershon MD, Ratcliffe EM (2004) Developmental biology of the enteric nervous system: pathogenesis of Hirschsprung disease and other congenital dysmotilities. Semin Pediatr Surg 13: 224-235

7. Heitz PU, Komminoth P (1990) Biopsy diagnosis of Hirschsprung's disease and related disorders. Curr Top Pathol 81: 257-275

8. Hess R, Scarpelli DG, Pearse AG (1958) The cytochemical localization of oxidative enzymes. II. Pyridine nucleotide-linked dehydrogenases. J Biophys Biochem Cytol 4: 753-760

9. Hirschsprung H (1889) Stuhlträgheit Neugeborener infolge von Dilatation und Hypertrophie des Colons. Jahrbuch der Kinderheilkunde 27: 1-7

10. Holschneider AM, Puri P (eds) (2000) Hirschsprung's disease and allied disorders, 2nd edn. Harwood Academic Publishers, Amsterdam, The Netherlands

11. Hüther W (1954) Die Hirschsprung'sche Krankheit als Folge einer Entwicklungsstörung der intramuralen Ganglien. Beitr Pathol Anat Allg Pathol 114: 161-191

12. Jacobi A (1869) On some important causes of constipation in infants. Am J Obstetrics 2:96-113

13. Kapur RP (1993) Contemporary approaches toward understanding the pathogenesis of Hirschsprung disease. Pediatr Pathol 13: 83-100

14. Karnovsky MJ, Roots L (1964) A, direct-coloring' thiocholine method for cholinesterases. J Histochem Cytochem 12: 219-221

15. Malone PS, Ransley PG, Kiely EM (1990) Preliminary report: the antegrade continence enema. Lancet 336: 1217-1218

16. Meier-Ruge WA (1965) Anwendung und Aussagewert der Fermenthistochemie in der speziellen und experimentellen Pathologie. Das Medizinische Laboratorium 18: 193-225

17. Meier-Ruge W (1967) Zur Pathologie und bioptischen Diagnostik des Morbus Hirschsprung. Verh Dtsch Ges Path 51: 327-328

18. Meier-Ruge WA, Morger R (1968) Neue Gesichtspunkte zur Pathogenese und Klinik des Morbus Hirschsprung. Schweiz Med Wochenschr 98: 209214

19. Meier-Ruge W (1968) Das Megacolon - Seine Diagnose und Pathophysiologie. Virchows Arch Pathol Anat 344: 67-85

20. Meier-Ruge W (1974) Hirschsprung's disease: ist aetiology, pathogenesis and differential diagnosis. Curr Topics Pathol 59: 131-179
21. Meier-Ruge W, Hunziker O, Tobler HJ, Walliser Ch (1972) The pathophysiology of aganglionosis of the entire colon (Zuelzer-Wilson-Syndrome). Beitr Path 147: 228-236

22. Meier-Ruge WA, Bruder E (2005) Pathology of chronic constipation in pediatric and adult coloproctology. Pathobiology 72: 1-102

23. Meier-Ruge WA, Bruder E (2005) Preparation of cryostat sections from biopsies and colorectal specimens. Pathobiology 72: 93-94

24. Okamoto E, Ueda T (1967) Embryogenesis of intramural ganglion of the gut and its relation to Hirschsprung's disease. J Pediatr Surg 2: 437-443

25. Swenson O, Bill AH (1948) Resection of rectum and rectosigmoid with preservation of the sphincter for benign spastic lesions producing megacolon: an experimental study. Surgery 24: 212-220

26. Tittel K (1901) Über eine angeborene Mißbildung des Dickdarms. Klin Wochenschr 14: 903-907

27. Whitehouse FR, Kernohan JW (1948) Myenteric plexus in congenital megacolon. Study of eleven cases. Arch Intern Med 82: 75-111

28. Zuelzer WW, Wilson JL (1948) Functional intestina obstruction of congenital neurogenic basis in infancy. Am J Dis Childhood 75: 40-64 